\title{
ANÁLISE DO DESEMPENHO DOS CANDIDATOS AOS CURSOS DA ANPEC, 1976-1982*
}

\author{
Silvio Aguiar*i \\ Antônio Aguirre*** \\ José Francisco Soares**
}

RESUMO

Neste artigo apresentamos os resultados da análise das notas dos candidatos aos cursos da ANPEC, obtidas nos concursos de selecão do período 1976-1982. Em primeiro lugar, discutimos sobre o nú mero de candidatos e sua relação com os três fatores de classificação utilizados na análise. Em segundo, analisamos as notas de quatro matérias dos concursos (Macroeconomia, Microeconomia, Estatística e Matemática) com referēncia aos mesmos fatores. Utilizamos Análise Fatorial para justificar a síntese dessas quatro notas em apenas duas (Teoria Econômica e Métodos Quantitativos), e Análi se de Variāncia Multivariada para estudar essas duas variáveis-res posta com relação aos trēs fatores de classificação. Procuramos identificar diferenças no nivel dos candidatos segundo o centro de pós-graduação que escolherem para ingresso, a cidade ou região do País onde fizeram prova, e o ano da mesma. Por último, verificamos possiveis interaçōes entre os fatores, e como subproduto obtivemos várias classificaçōes dos centros.

Os autores agradecem o apoio da ANPEC, do Conselho de Pesquisas da UFMG, e do Centro de Computação (CECOM) da UFMG.

*** Departamento de Estatistica - Universidade Federal de Minas Gerais.

**** Centro de Desenvolvimento e Planejamento Regional; Faculdade de Ciências Econômicas; Universidade Federal de Minas Gerais. 


\section{ABSTRACT}

The National Association of Postgraduate Studies in Economics (ANPEC) administers yearly the entrance examination for candidates all over Brazil. In this paper we report the main results of an statistical analysis of the grades obtained by candidates in the period 1976-1982. The grades correspond to four subjects: Macroeconomics, Microeconomics, Statistics and Mathematics. We used Fac tor Analysis to justify the summarization of those four grades into two, namely Economic Theory and Quantitative Methods. We also used Multiple Analysis of Variance to study those two response variates in relation to three factors of classification. In addition to trying to identify differences in the candidates' levels with reference to the same factors, we investigated possible interactions a mong the factors, and produced several classifcations of the centers. 
1. INTRODUCAOO

A Associação Nacional de Centros de Pós-Graduação em Economia (ANPEC) foi fundada em janeiro de 1973, com a finalidade de congregar as instituiçōes brasileiras que operam no campo da Econo mia, através de pesquisas e formação de pessoal especializado a ní vel de pós-graduação.

Uma atividade tradicional da ANPEC é a organização dos concursos anuais para seleção dos candidatos aos diferentes cursos de pós-graduação em Economia oferecidos nos centros membros da Associação. Estes concursos constam de seis provas: Macroeconomia, Estatística, Matemática, Microeconomia, Economia Brasileira e Inglēs.

No segundo semestre de 1981, iniciou-se no Centro de Desenvol vimento e Planejamento Regional (CEDEPLAR) da UFMG um esforço de compilação e organização de todas as informaçōes disponiveis sobre os concursos da ANPEC. Isso só pôde ser feito com os concursos rea lizados a partir de 1976, pois só desde então as notas obtidas e outras informaçōes de cada candidato foram conservados. 0 objetivo deste esforço era realizar análises estatisticas desses dados, cujos resultados fossem ūteis às várias escolas de Economia do Brasil.

A primeira tarefa realizada foi a formação de um arquivo contendo todos os dados disponiveis. Terminada esta etapa foram prepa rados alguns trabalhos descritivos, focalizando diferentes aspectos dos dados, com a finalidade dupla de conferir a consistēncia dos mesmos e divulgá-los entre os possiveis interessados neste assunto (Aguirre, 1983).1/

1 As referēncias constam ao final do trabalho. 
Neste artigo procuramos identificar diferenças no nível dos candidatos segundo o centro que escolheram para ingresso, a cidade ou regiāo do País onde fizeram prova, e o ano da mesma. Verificamos, também, possíveis interações entre estes fatores, e, como sub produto, obteremos várias classificações entre os centros.

2. DESCRICATO DOS DADOS E NOTACÃO

o produto final da fase de compilação consistiu em um arquivo onde, para cada candidato que prestou o concurso, temos: a nota obtida em cada prova, a cidade onde fez o concurso, e os nomes de dois centros de pós-graduação, classificados por ordem de prefe rência, onde ele gostaria de ser admitido.

Temos portanto trēs fatores de classificação dos candidatos: ANO, com 6 níveis (1976-1981); primeira opção, que denotaremos sim plesmente por OPÇÃ, com 12 níveis:

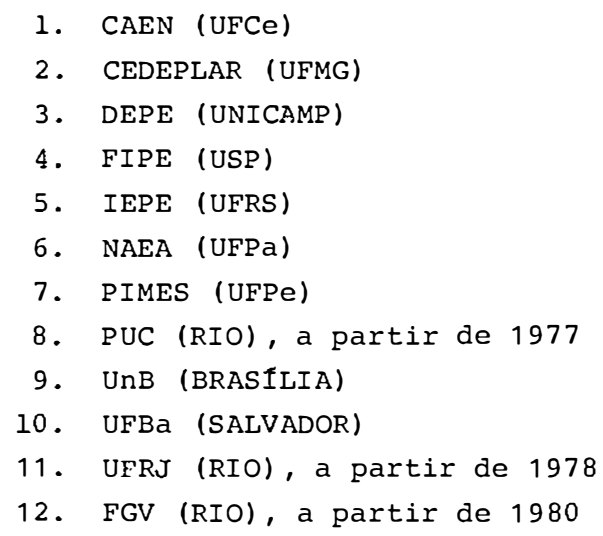

2. CEDEPLAR (UFMG)

3. DEPE (UNICAMP)

4. FIPE (USP)

5. IEPE (UFRS)

6. NAEA (UFPa)

7. PIMES (UFPe)

8. PUC (RIO), a partir de 1977

9. UnB (BRASILIA)

10. UFBa (SALVADOR)

11. UFRJ (RIO), a partir de 1978

12. FGV (RIO), a partir de 1980

Por último, usaremos o local de prova como uma aproximação pâ ra a origem do candidato, já que não há informação sobre a escola por ele frequentada. Este fator a ser denotado por ORIGEM tem 9 niveis: 
1. Belo Horizonte

2. Brasilia

3. Campinas

4. Porto Alegre

5. Rio de Janeiro

6. Salvador

7. São Paulo

8. Nordeste, onde estão incluídos todos os centros do Nordes te do País, exceto Salvador.

9. Outros.

Temos, portanto, $6 \times 12 \times 9=648$ combinações possíveis - a serem chamadas de celas - onde poderíamos alocar os candidatos. Des tas, as 108 correspondentes ao ano de 1976, não puderam ser preenchidas já que para aquele ano a informação sobre o local de prova dos candidatos não foi conservada, e 197 outras estão vazias.

o Quatro 1 permite acompaniar a evolução do tamanho da clientela da Associação. O número de candidatos ausentes em 1976 não é conhecido.

Quadro 1

NÜMERO DE CANDIDATOS NOS CONCURSUS DA ANPEC $1976 / 82$

\begin{tabular}{c|c|c|c}
\hline ANO & PRESENTES & AUSENTES & TOT AL \\
\hline 1976 & 251 & - & - \\
1977 & 408 & 86 & 494 \\
1978 & 83 & 577 \\
1979 & 994 & 57 & 512 \\
1980 & 415 & 507 \\
1981 & 437 & 55 & 444 \\
1982 & 408 & 463 \\
\hline
\end{tabular}

FONTE: ANPEC. 


\section{NUMERO DE CANDIDATOS E OS FATORES DE CLASSIFICACÃO}

Os Quadros 2, 3 e 4 nos dão os totais de candidatos segundo os três possiveis cruzamentos, dois a dois, entre os fatores de classificação antes definidos.

Quadro 2

NUNERO DE CANDIDATOS POR ANO E ORICEM

\begin{tabular}{l|r|r|r|r|r}
\hline \multicolumn{1}{c}{ ORIGEM } & \multicolumn{5}{|c}{ A N } \\
\cline { 2 - 6 } & 1977 & 1978 & 1979 & 1980 & 1981 \\
\hline Beio Horizonce & 33 & 47 & 49 & 25 & 19 \\
Brasilia & 54 & 53 & 46 & 79 & 65 \\
Campinas & 19 & 34 & 18 & 25 & 29 \\
Porto Alegre & 26 & 21 & 18 & 10 & 14 \\
Rio de Janeiro & 80 & 106 & 100 & 121 & 86 \\
Salvador & 24 & 28 & 25 & 17 & 29 \\
Säo Paulo & 78 & 98 & 100 & 91 & 89 \\
Nordeste & 56 & 64 & 34 & 36 & 15 \\
Outros & 38 & 43 & 25 & 33 & 32 \\
\hline
\end{tabular}

FONTE: ANPEC.

Quadro 3

NOMERO DE CANDIDATOS POR ANO E OPCXO

\begin{tabular}{|c|c|c|c|c|c|c|}
\hline \multirow{2}{*}{ OPCĀO } & \multicolumn{6}{|c|}{$A N O$} \\
\hline & 1976 & 1977 & 1978 & 1979 & 1980 & 1981 \\
\hline CAEN (UFCe) & 13 & 31 & 25 & 21 & 21 & 14 \\
\hline CEDEPLAR (UFMG) & 39 & 52 & 56 & 41 & 24 & 16 \\
\hline DEPE (UNICAMP) & 75 & 67 & 85 & 63 & 63 & 49 \\
\hline FIPE (USP) & 51 & 73 & 74 & 88 & 77 & 92 \\
\hline IEPE (UFRS) & 8 & 22 & 22 & 20 & 8 & 8 \\
\hline NAEA (UFPa) & 2 & 10 & 4 & 5 & 5 & 11 \\
\hline PIMES (UFPe) & 14 & 21 & 30 & 17 & 18 & 6 \\
\hline $\mathrm{PUC} / \mathrm{RJ}$ & - & 75 & 60 & 52 & 30 & 16 \\
\hline UnB (BKASILIA) & 36 & 38 & 42 & 35 & 42 & 47 \\
\hline UFBa (SALVADOR) & 13 & 19 & 24 & 19 & 12 & 25 \\
\hline UFR J & - & - & 72 & $\therefore$ & 47 & 39 \\
\hline EPGE / FGV & - & - & - & - & 90 & 55 \\
\hline
\end{tabular}

FON'TE: ANPEC. 
Quadro 4

NÚMERO DE CANDIDATOS POR ORIGEM E OPÇÃO

\begin{tabular}{l|c|r|r|r|r|r|r|r}
\hline & $\begin{array}{c}\text { Belo } \\
\text { Horizonte }\end{array}$ & Brasilia & Campinas & $\begin{array}{r}\text { Porto } \\
\text { Alegre }\end{array}$ & Rio & Salvador & $\begin{array}{c}\text { São } \\
\text { Paulo }\end{array}$ & Nordeste \\
\hline CEDEPLAR & 107 & 13 & 3 & 4 & 12 & 3 & 7 & 13 \\
UnB & 5 & 144 & 4 & 2 & 6 & 2 & 7 & 11 \\
UNICAMP & 13 & 17 & 85 & 15 & 30 & 14 & 96 & 24 \\
UFRS & 3 & 4 & 1 & 49 & 3 & 3 & 1 & 8 \\
Rio & 27 & 70 & 10 & 7 & 408 & 8 & 16 & 16 \\
UFBa & 2 & 5 & 1 & 3 & 0 & 78 & 3 & 5 \\
FIPE/USP & 4 & 26 & 17 & 6 & 15 & 6 & 304 & 11 \\
Nordeste & 10 & 13 & 4 & 2 & 16 & 5 & 17 & 114 \\
\hline
\end{tabular}

FONTE: ANPEC.

Um teste do $\mathrm{x}^{2}$, a um nível de significância de 1\%, nos mostra que a evolução do número de candidatos tem padrões diferentes nas vārias origens. Por exemplo, Belo Horizonte perdeu candidatos e Brasilia ganhou, enquanto que em Campinas o número permaneceu está vel. Deve-se notar ainda a drástica queda, em 1981, no número de candidatos originārios do Nordeste (ver Gráfico 1). 


\section{Gráfico 1}

NÚMERO DE CANDIDAT OS POR ANO E ORIGEM

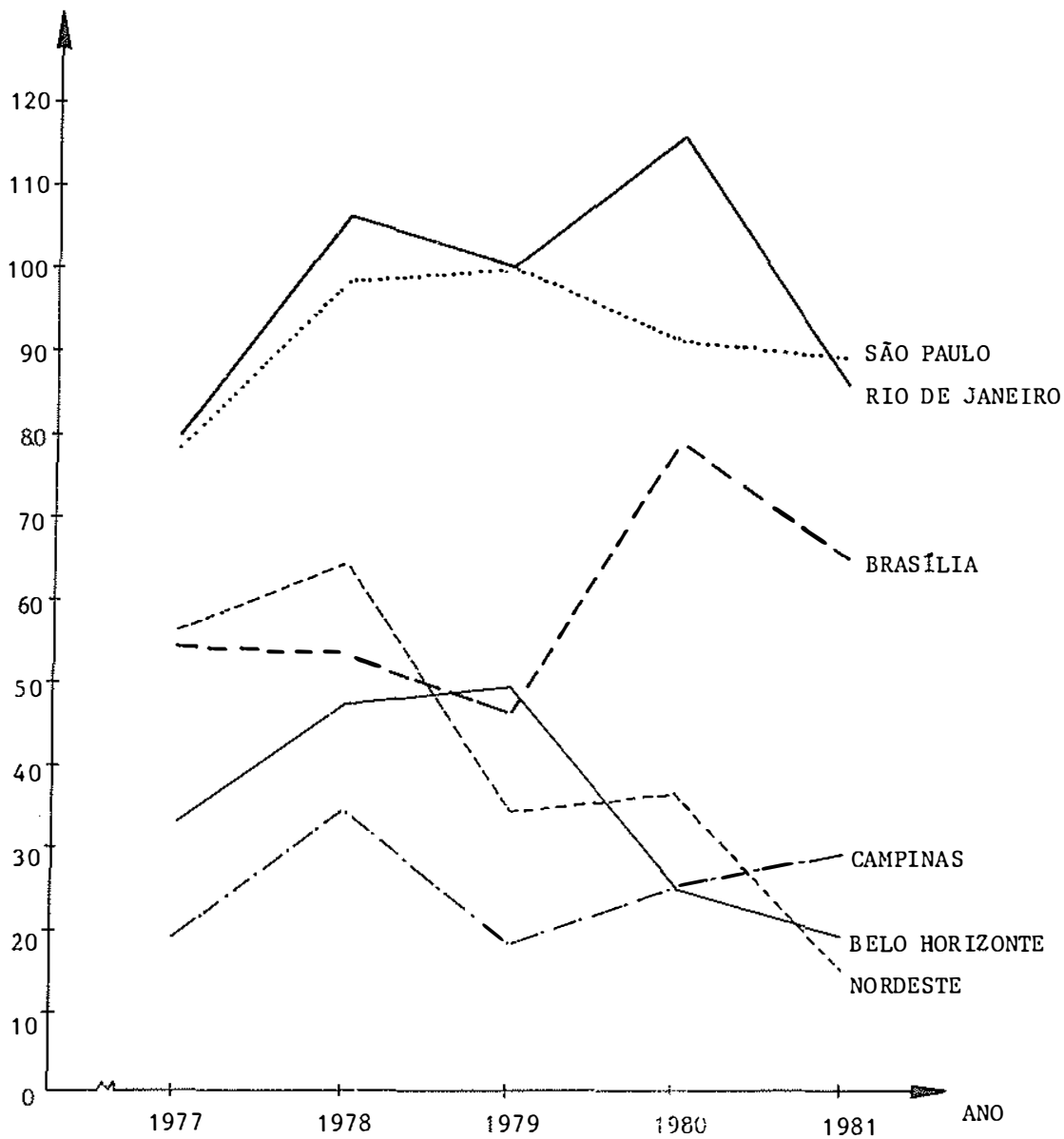

Fonte: Quadro 2 . 
Deve-se chamar a atenção para a inclusão, a partir de 1980, da FGV como nova opção. Nota-se que, nos dois anos em que funcionou, esse Centro foi um dos mais procurados; ou seja, com a inclusão da FGV as opções abertas aos candidatos aumentaram mais do que a simples adição de mais um centro poderia levar a supor (Quadro 3).

Para construir o Quadro 4, que nos dá o número de candidatos por ORIGEM e OPÇÃO tivemos de reduzir o número de categorias no critērio OPÇÃO. As opções UFPe e UFCe foram agrupadas numa catego ria chamada "Nordeste", e as opções UFRJ, PUC/RJ e FGV formaram outra denominada "Rio". A categoria "outros" no fator ORIGEM e o NAEA (UFPa) no fator OPÇÃo foram excluídas do Quadro 4.

E interessante notar a forte tendência dos candidatos a escolher um Centro de pós-graduação da própria cidade de origem, haja visto a concentração do número de candidatos na diagonal principal do Quadro 4. A OPÇÃO UNICAMP, entretanto, é a mais procurada (proporcionalmente) por candidatos de todas as origens, o qual pode ser o reflexo do renome desse Centro entre os candidatos de todo o Pais.

4. NOTAS DE TEORIA ECONOMMICA E METCDOS QUANTITATIVOS

Uma preocupação dos professores ligados à pós-graduação tem sido o nível de conhecimento nas matérias básicas que o formando em Economia possui. As análises desta e das próximas seções descre vem com detalhes as notas e, enquanto elas possam ser consideradas medidas confiáveis, o nivel dos formandos que optaram por fazer mes trado.

A escala utilizada para as notas tem um teto de 10, mas, dado que o sistema adotado penaliza os erros, tentando com isso evitar respostas aleatórias, é possível observar-se notas negativas. Deve -se notar, primeiramente, o baixo valor das notas em geral. Detalhes disto estão em (Aguirre, 1983). 
É razoável admitir que as quatro notas dos candidatos possam ser sintetizadas em duas: uma de Teoria Econômica, formada pela mẹ dia das notas de Macroeconomia e Microeconomia, e outra de Métodos Quantitativos, constituída pela média das notas de Estatística e Matemática.

A técnica estatistica chamada Análise Fatorial legitima esta sintese. Utilizando esta técnica verificamos que se passarmos a tra balhar com as seguintes combinaçōes lineares:

$$
\begin{aligned}
& F_{1}=-0,28 x_{1}+0,67 x_{2}+0,55 x_{3}+0,02 x_{4} \\
& F_{2}=0,84 x_{1}-0,35 x_{2}-0,17 x_{3}+0,49 x_{4}
\end{aligned}
$$

onde: $x_{1}=$ Macroeconomia; $x_{2}=$ Estatistica; $x_{3}=$ Matemática $e$ $x_{4}=$ Microeconomia, estaremos preservando $80 z$ da variabilidade existente nos dados e explicando bastante bem a estrutura de correlação entre as notas.

Um resumo dos resultados obtidos na aplicação desta técnica è dado pelo Gráfico 2 .

$$
\text { Gráfico } 2
$$

RESULTADOS DA A.NELISE FATORIAL

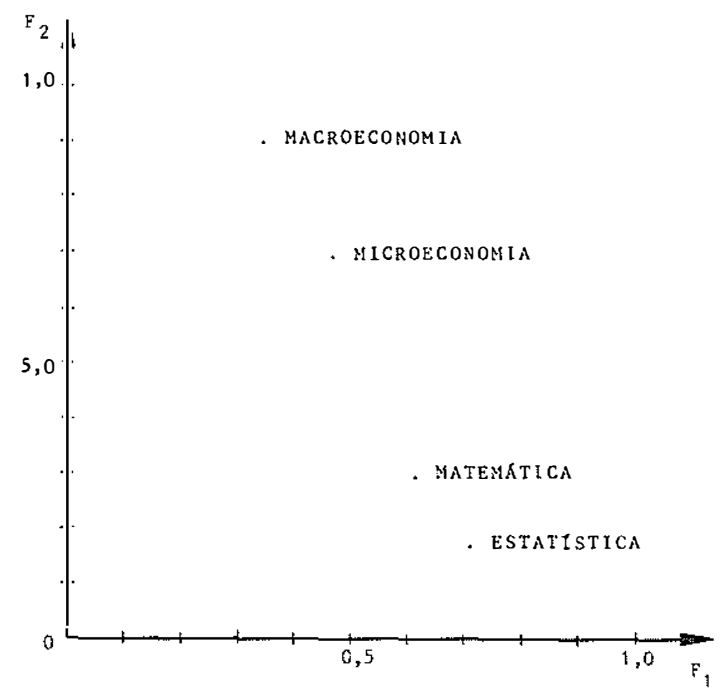


Os dois eixos correspondem às combinações lineares $F_{1}$ e $F_{2}$. Observe-se que as matérias Estatística e Matemática estão bem próximas do primęiro eixo, Macro está bem próximo do segundo, e Micro, ainda que próxima do segundo, tende a ficar no meio. Em função disso poderíamos resumir as notas por essas duas combinações lineares. En tretanto, como as médias Teoria Econômica e Métodos Quantitativos são uma boa aproximação para $\mathrm{F}_{1}$ e $\mathrm{F}_{2}$, e possuem fácil interpretação, de ora em diante usaremos estas duas médias na maioria das anālises. Isto facilitará principalmente a construção de gráficos, jā que podemos trabalhar com apenas duas dimensões.

\section{AS NOTAS E OS FATORES DE CLASSIFICACAOO}

Nesta seção mostraremos, isoladamente, o efeito de cada fator de classificação nas notas dos candidatos.

5.1. O Fator ANO

O Quadro 5 apresenta as médias das notas dos candidatos nos vārios anos para as quatro disciplinas. A apresentação é um pouco diferente do usual: no corpo da tabela aparecem os resíduos, depois de retirados os efeitos da média geral, das colunas e das linhas. Os valores destes efeitos aparecem circundando a tabela. Por exemplo, a média de Macroeconomia, em 1976, foi $6,09=2,00+2,86$ $+(-0,15)+1,38$ ou seja: Resíduo + Média Geral + Coluna + Linha. 
Quadro 5

RESIDUOS APOS RETIRADA DOS EFEITOS ANO E MATERIA

\begin{tabular}{c|c|c|c|c|c}
\hline ANO & MACRO & ESTATISTICA & MATEMATICA & MICRO & IINHA \\
\hline 1976 & 2,00 & $-1,34$ & $-0,39$ & $-0,29$ & 1,38 \\
1977 & $-0,30$ & $-0,09$ & $-0,09$ & 0,48 & $-0,53$ \\
1978 & $-0,02$ & $-0,03$ & $-0,12$ & 0,15 & 0,07 \\
1979 & $-0,50$ & 0,87 & $-0,24$ & $-0,12$ & $-0,22$ \\
1980 & $-0,52$ & 0,44 & 0,71 & $-0,63$ & $-0,60$ \\
1981 & 0,18 & 0,21 & $-0,45$ & 0,47 & $-0,27$ \\
1982 & $-0,87$ & 0,36 & 0,60 & 0,09 & 0,17 \\
\hline COLUNA & $-0,15$ & $-0,11$ & 0,38 & $-0,12$ & 2,86 \\
\hline
\end{tabular}

Os resíduos do Quadro 5 são os que seriam obtidos ajustando-se às médias anuais das notas um modelo de Análise de Variância com dois fatores. As médias estão classificadas pelos fatores matê rias e ano. o valor dos resíduos nos chama atenção para cinco provas: como provas fáceis Macro 76, Estatística 79, e Matemática 80 ; como dificeis Estatistica 76, Macro 82 e Micro 80 . 0 uso dos adjetivos "fácil - difícil" é um tanto subjetivo já que não temos como saber se a variação observada nas médias é devida à variação na qualidade dos candidatos de ano para ano ou à variação na dificuldade das provas.

5.2. O Fator ORIGEM

o Quadro 6 mostra as médias das notas dos candidatos com a mes ma origem. O Gráfico 3 é um sumārio esclarecedor desse quadro. 
Quadro 6

MEEDIA DAS NOTAS POR ORIGEM DO CANDIDATO

\begin{tabular}{|c|c|c|c|c|}
\hline & MACRO & ESTATISTICA & MATEMATICA & MICRO \\
\hline 1. Belo Horizonte & 2,19 & 2,47 & 2,93 & 2,43 \\
\hline 2. Brasilia & 2,05 & 2,49 & 2,87 & 2,64 \\
\hline 3. Campinas & 2,82 & 2,97 & 3,30 & 2,74 \\
\hline 4. Porto Alegre & 1,87 & 2,01 & 1,74 & 1,79 \\
\hline 5. Rio & 2,76 & 3,51 & 3,85 & 3,04 \\
\hline 6. Sa lvador & 1,38 & 1,10 & 1,87 & 1,86 \\
\hline 7. São Paulo & 2,36 & 3,13 & 3,13 & 2,72 \\
\hline 8. Nordeste & 1,35 & 1,84 & 2,35 & 1,86 \\
\hline 9. Outros & 1,46 & 1,51 & 1,43 & 1,56 \\
\hline
\end{tabular}

Gräfico 3

NOTAS EM TEORIA ECONMICA

F. MÉTODOS QUANTITATIVOS PELA ORIGEM

MÉTODOS

QUANT ITATIVOS

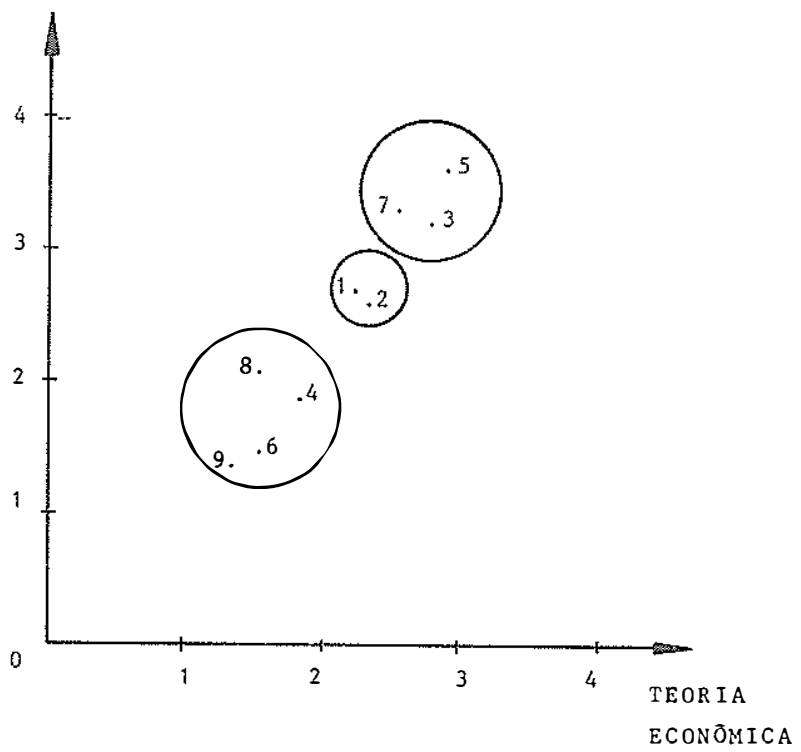


Fica claro que as origens podem ser agrupados em termos de qualidade dos candidatos em trēs grupos: 1. São Paulo, Campinas, Rio; 2. Belo Horizonte, Brasilia; 3. Nordeste, Salvador, Porto Ale gre. A qualidade dos candidatos em Teoria Econômica está intimamen te associada à qualidade em Métodos Quantitativos. Merece destaque a média dos candidatos do Rio em Métodos Quantitativos. Seria interessante verificar quem tem feito as questões para as provas e a orientação e conteúdo das mesmas e associar estas observações com o observado no Quadro 6 .

\section{3. O Fator OPCÁO}

Agora podem ser feitas observações parecidas com aquelas apre sentadas para o fator ORIGEM. Os grupos formasos são: 1. UFRJ, PUC/RJ, USP e UNICAMP; 2.FGV/RJ, UFMG e UnB; 3. UFRS, UFPe, UFBa, UFCe e UFPa. A posição da FGV/RJ é singular: os candidatos que a procuram têm tido em média excelente nível em Métodos Quantitativos e nível apenas sofrivel em Teoria Econōmica. De novo aparece claramente à associação entre os dois fatores:os candidatos cpuando bons, o são nos dois fatores. A UNICAMP é procurada pelos candidatos que em média têm melhor nível em Teoria Econômica, a UFRJ atrai os melhores candidatos em Métodos Quantitativos. Essas afirmativas são reexaminadas nas próximas seções (ver Quadro 7 e Gráfico 4).

Quadro 7

MEDIA DAS NOTAS POR OPGAO DO CANDIDATO

\begin{tabular}{ll|c|c|c|c}
\hline & & MACRO & ESTATISTICA & MATEMATICA & MICRO \\
\hline 1. UFCe & 1,86 & 2,18 & 2,49 & 2,03 \\
2. UFMG & 2,54 & 2,18 & 2,77 & 2,45 \\
3. UNICAMP & 3,37 & 3,00 & 3,45 & 3,14 \\
4. USP & 2,75 & 2,93 & 3,23 & 2,69 \\
5. UFRS & 2,15 & 1,68 & 1,73 & 1,94 \\
6. UFPa & 1,20 & 1,17 & 1,72 & 1,44 \\
7. UFPe & 1,86 & 1,57 & 1,92 & 2,05 \\
8. PUC/RJ & 2,57 & 3,30 & 3,61 & 2,99 \\
$9 . \quad$ UnB & 2,47 & 2,41 & 2,79 & 2,75 \\
10. UFBa & 1,81 & 1,29 & 2,01 & 1,9 \\
$11 . \quad$ UFRJ & 2,83 & 3,61 & 3,63 & 3,13 \\
12. FGV & 2,25 & 2,94 & 3,82 & 2,13 \\
\hline
\end{tabular}


Gräfico 4

NOTAS EM TEORIA ECONOMICA E METTODOS

QUANTITATIVOS POR OPÇÃO

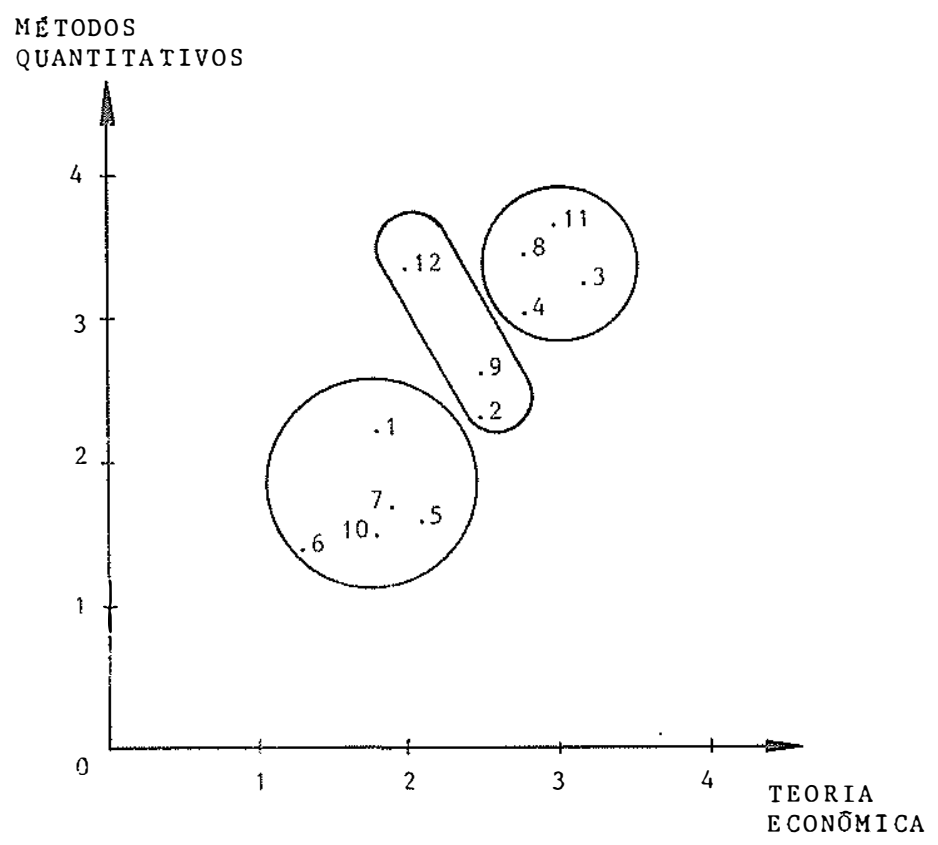

Embora os resultados obtidos sejam interessantes, o ideal seria considerar, na classificação dos centros, a média dos alunos realmente admitidos, e não a dos que simplesmente optaram. Infeliz mente isto não é possivel com os dados disponíveis.

6. ANALLISE MULTIVARIADA

Embora informativas, as anālises anteriores têm uma séria limitação: apenas um fator é consideracio de cada vez, enquanto que, na realidade, os fatores ANO, OPCAO e ORIGEM determinam conjunta- 
mente as notas dos candidatos. E, portanto, interessante perguntar mo-nos se as afirmações da Seção 5 se modificam se levarmos em con sideração os outros fatores. Em termos estatisticos estamos interessados em verificar a existência de interações entre os fatores. Tal estudo deveria ser feito ajustando-se aos dados o modelo estatístico apropriado, qual seja a Análise de Variância Multivariada, já que são duas as variāveis resposta, i.e., as notas em Teoria Econômica e em Métodos Quantitativos, e trēs os fatores de classificação. Entretanto, esse modelo não pode ser ajustado aos nossos dados, devido ao grande número de celas vazias e à limitação do "software" estatístico disponivel.

Para contornar essas dificuldades trabalhamos com as seguintes simplificações: consideramos uma resposta de cada vez, limitan do-nos aos modelos de anālise de variância usuais, e reduzimos o número de categorias dos fatores. Para o fator ANO, excluímos 1976 já que a informação sobre o local de prova não foi conservada para este ano; para o fator ORIGEM excluímos as categorias Porto Aiegre, Salvador, Nordeste e Outros; e para o fator OPÇÃO agrupamos as escolas ḋo Rio de Janeiro em uma única categoria, e deixamos de considerar UFCe, UFRS, UFPa, UFPe e UFBa. AO fazer estরis ejclusões pio curamos reduzir consideravelmente o número de celas vazias, manten do o maior número possível de candidatos. Os Quadros 8 e 9 sumariam os resultados do ajuste do modelo de análise da variāncia.

Notamos primeiramente o pequeno poder explicativo dos três fatores considerados. O coeficiente de determinação, que seria 100\% caso os fatores explicassem completamente as notas dos candidatos, é 19,598 para Teoria Econômica e 13,86\% para Métodos Quantitativos. Esses baixos valores nos dizem que para se entender a variabi lidade das notas dos candidatos é preciso considerar outros fatores além dos trēs aqui incluídos. O potencial individual de cada candidato é, por exemplo, um fator com jbvio poder explicativo, que não pode ser considerado neste trabalho por falta de dados. 
Quadro 8

ANÁLISE DA VARIÁNCIA PARA TEORIA ECONOMICA

\begin{tabular}{|c|c|c|c|c|c|}
\hline FONTES DE VARIAÇÃO & $\begin{array}{c}\text { SOMA DE } \\
\text { QUADRADOS } \\
\end{array}$ & G. & $\begin{array}{c}\text { QUADRADOS } \\
\text { MEDIOS } \\
\end{array}$ & $\mathrm{F}$ & $\begin{array}{l}\text { SIGNIF ICÃNCIA } \\
\text { DO TESTE }\end{array}$ \\
\hline Efeitos Principais & 330,706 & 12 & 27,559 & 9,845 & 0,000 \\
\hline ANO & 277,163 & 4 & 69,291 & 24,752 & 0,000 \\
\hline OPÇÃO & 38,641 & 4 & 9,660 & 3,451 & 0,008 \\
\hline ORIGEM & 26,089 & 4 & 6,522 & 2,330 & 0,054 \\
\hline Interação 2 a 2 & 277,493 & 48 & 5,781 & 2,065 & 0,000 \\
\hline ANO X OPÇÃO & 71,058 & 16 & 4,441 & 1,586 & 0,065 \\
\hline ANO $X$ ORIGEM & 140,208 & 16 & 8,763 & 3,130 & 0,000 \\
\hline OPÇÃO X ORIGEM & 55,505 & 16 & 3,469 & 1,239 & 0,230 \\
\hline Explicada & 944,452 & 60 & 15,741 & 5,623 & 0,000 \\
\hline Residual & $3.877,144$ & 1.385 & 2,799 & & \\
\hline $\mathrm{T} O \mathrm{TAL}$ & $4.821,597$ & 1.445 & 3,337 & & \\
\hline
\end{tabular}

Quadro 9

ANALISE DA VARIÂNCIA PARA METODOS QUANTITATIVOS

\begin{tabular}{l|r|r|r|c|c}
\hline \multicolumn{1}{|c|}{ FONTES DE VARIAÇÃO } & SOMA DE & G.L. & $\begin{array}{r}\text { QUADRADOS } \\
\text { QUADRADOS }\end{array}$ & F & $\begin{array}{r}\text { SIGNIFICÃNCIA } \\
\text { DO }\end{array}$ \\
\hline EfEST E
\end{tabular}


A ūltima coluna dos Quadros 8 e 9 nos mostra a significância estatística dos termos na Análise de Variâncià. Quanto mais próxima de zero é a significância, mais significativo é o termo. observamos, portanto, uma clara interação entre os fatores ANO e ORIGEM, tanto para Teoria Econômica como para Métodos Quantitativos. Isto quer dizer que o par ANO e ORIGEM influencia conjuntamente o nivel dos alunos. Por isso não podemos afirmar, por exemplo, que os candidatos originários de Campinas sejam melhores que os de Belo Hori zonte, apesar que todas as médias de Campinas sejam maiores que as correspondentes de Belo Horizonte (ver Quadro 6). Tal comparaçãosó pode ser feita dentro de um determinado ano.

Os resultados da Seção 5 são portanto limitados, já que não levam em consideração essa ação conjunta. Vários fatos interessantes não apareceram portanto naquelas análises. Essas especificida des podem ser descobertas através de gráficos apropriados. Por exemplo, o Gráfico 5 foi construido da mesma forma que o Gráfico 4, usando-se entretanto apenas os candidatos com origem em Belo Horizonte. Esses dois gráficos são bastantes parecidos, os Centros estão aproximadamente na mesma posição, com uma notável exceção: os 5 estudantes de Belo Horizonte que procuraram Brasilia foram melho res, em média, que os de outras origens que procuraram Brasilia. Gráficos semelhantes foram feitos para outras origens, e não são apresentados por questão de espaço. Os gráficos para ANO e OPÇÃO realçaram o excelente desempenho dos candidatos que procuraram a PUC/RJ em 1981.

Outro tipo de gráfico tastante elucidativo do fenômeno da interação é o Gráfico 6. Cada ponto ali nos dá a média em Teoria Eco nômica para os candidatos de uma dada origem, num dado ano. A inte ração entre os fatores ORIGEM e ANO constatada no quadro de análise da variāncia é vista aqui através do não paralelismo das linhas correspondentes aos vários anos. Neste gráfico é de se notar o fra co desemepenho dos candidatos originários de Campinas em 1979, o bom desempenho geral dos candidatos de todos os centros em 1978 , e o baixo desempenho dos mesmos em 1980 . 


\section{Gràfico 5}

NOTAS EM TEORIA ECONOMICA E METODOS QUANTITATIVOS, DOS CANDIDATOS DE BELO HORIZONTE, POR OPÇÃO

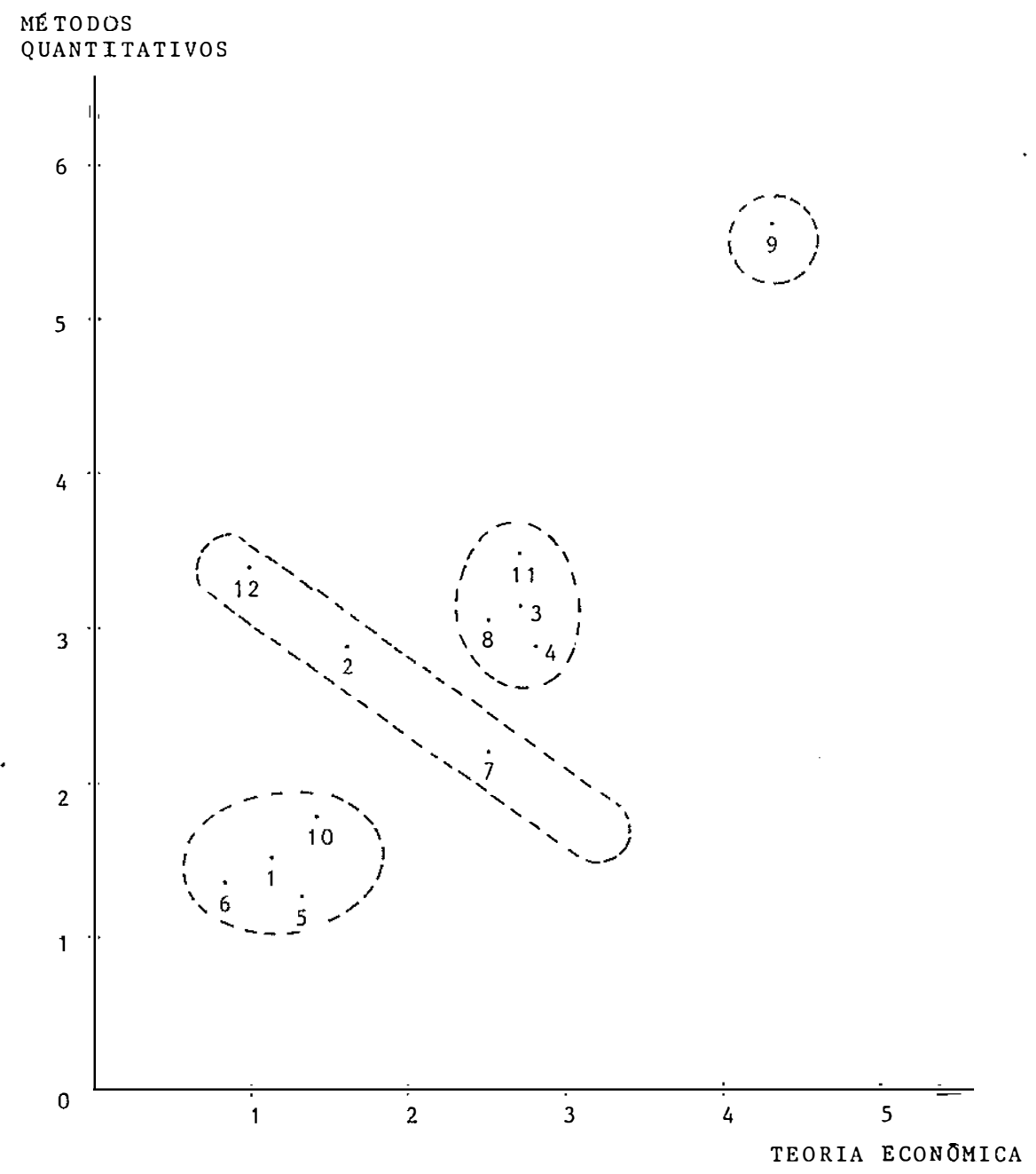




\section{Grafico 6}

INTERAÇÃO DA ORIGEM VERSUS ANO EM TEORIA ECONŌMICA

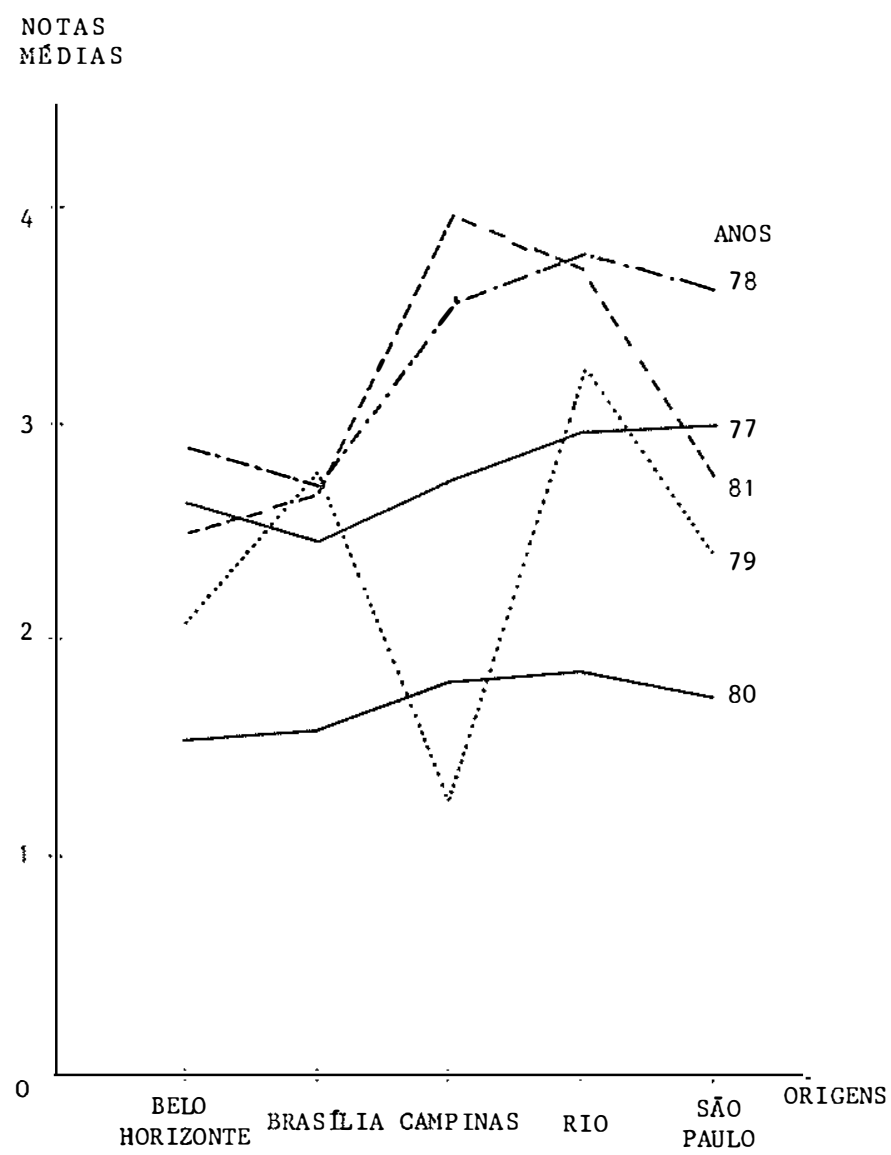


- Grā́ico 7, construído do mesmo modo, mostra a interação entre a ORIGEM e ANO para Métodos Quantitativos. Além do não paralelismo das linhas correspondentes aos vărios anos, o que nos forne ce um indício da significância da interação, devemos também notar a inclinação das linhas em relação à localização dos fatores na ho rizontal, que no Gräfico 7 são as origens. Essa inclinação indica a influência das origens nas notas, fatoque já fora constatado pelo valor da estatística $F$ para o efeito principal do fator ORIGEM, no Quadro 9. Nota-se, por exemplo, a especial influência das origens nas notas de 1979.

Gräfico 7

INTERAÇÃO DA ORIGEM VERSUS ANOS EM METODOS QUANTITATIVOS

NOTAS

MẼD IA S

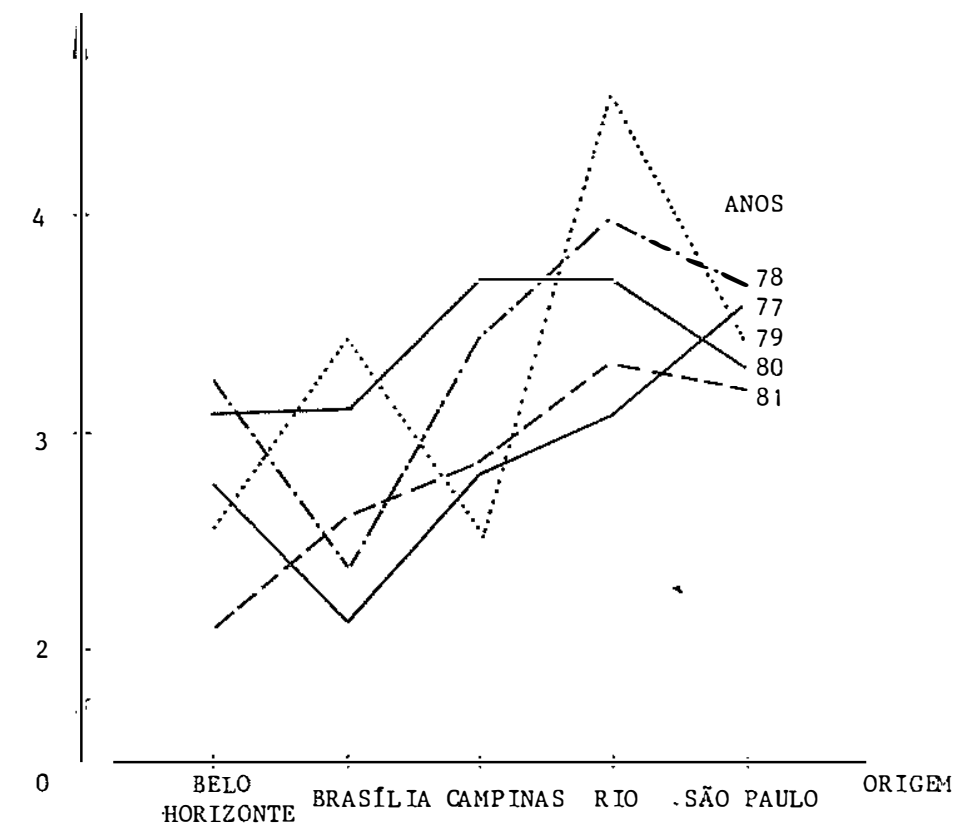


O Grāfico 8 ilustra a interação entre OPÇÃO e ANO em Teoria $\because$ 'onômica. Além da inclinação das linhas em relação à localização uas opçōes, devemos, também, notar as distâncias entre as poligorais. A distância entre duas poligonais quaisquer indica o efeito $\because$ diversos anos nas notas. A maior distância entre as poligonais do: anos 1:78 e 1980, por exemplo, é um indício intuitivo do efeito dos dilos nas notas de Teoria Econômica.

Gräfico 8

INTERAÇÃO DA OPÇÃO VERSUS ANOS EM TEORIA ECONŌMICA

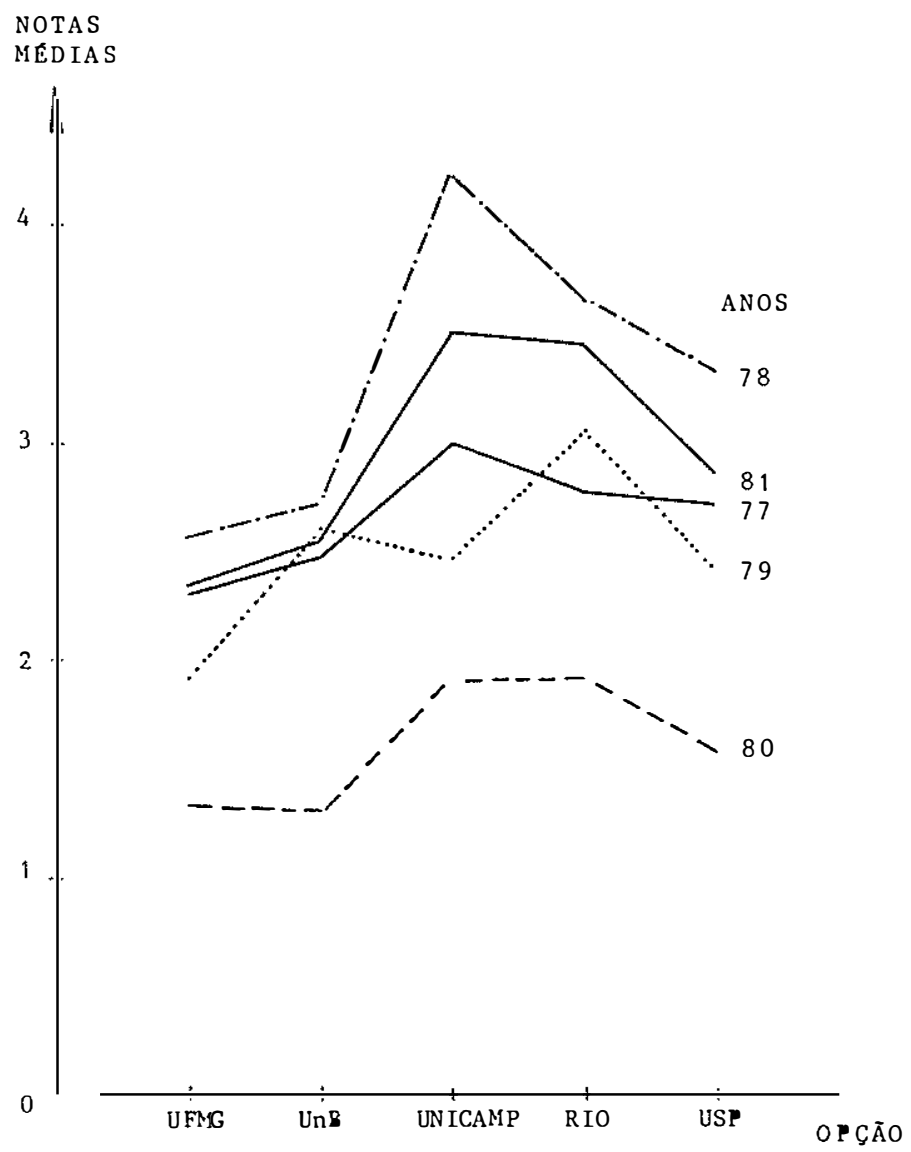




\section{COMPARACOES ENTRE MEDIAS}

Nos Gráficos 6,7 e 8 os pontos estão baseados em números diferentes de observações e, em alguns casos, esse número é bastante pequeno. Por isso, as diferenças observadas podem não ser significantes. Isso pode ser verificado através de comparações de médias. A constatação, feita na seção anterior, da existēncia de interações entre ANO X ORIGEM e ANO X OPÇÃO, para Teoria Econômica, e ANO X ORIGEM para Métodos Quantitativos, nos obriqa a considerar a ação conjunta dos fatores nas comparações de médias.

Por exemplo, o procedimento para responder à pergunta: "são os candidatos que optaram pelo CEDEPLAR, melhores em Teoria Econômica que aqueles que optaram por Brasilia?", é o seguinte. Como há interação entre ANO e OPÇÃO, a pergunta tem respostas, diferentes para anos diferentes. Para cada ano devem-se considerar os candida tos de todas as origens que optaram pelo CEDEPLAR e Brasilia, e construir um intervalo de confiança para a diferença de médias usando os limites preconizados por Bonferroni (Neter et. al., 1974).

Na seqüência verificaremos a significância de algumas diferen ças sugeridas pelos Gráficos 6, 7 e 8 . Em primeiro lugar, testaremos as diferenças, ao longo dos anos, das notas médias de Teoria $\underline{\mathrm{E}}$ conômica, para os candidatos originários de Campinas, cuja existên cia é sugerida pelo Grafíco 6. Depois, baseados no Gráfico 8, veri ficaremos as diferenças das notas médias de Teoria Econômica, ao longo dos anos, para os candidatos que optaram pela UNICAMP. Compa raremos ainda as notas médias de Métodos Quantitativos, ao longo dos anos, para os candidatos originārios de Rio de Janeiro (ver Gráfico 7). Naturalmente, muitas outras comparações sugeridas por esses grāficos poderiam ser feitas. Além do mais, outros gráficos semelhantes aos apresentados poderiam ter sido construidos, os quais sugeririam outras comparações. Desse conjunto apresentaremos, pela sua generalidade, a comparação das opções em Métodos Quan titativos. 
7.1. As notas de Teoria Econömica, ao longo dos anos, na ORIGEM Campinas e na OPCAO UNICAMP

O Quadro 10 permite concluir que, a um nivel de confiança de 95:, os desempenhos dos candidatos da ORIGEM Campinas nos anos de 1979 e 1980 são equivalentes, mas inferiores ao de 1977, o qual, por sua vez, é inferior ao de 1978 e 1981 que também são estatistí camente iguais. Portanto, nem todas as diferenças sugeridas pelo Gráfico 6 para a ORIGEM Campinas são significantes.

Quadro 10

COMPARAÇÃO DAS NOTAS MEDIAS DE TEORIA ECONONMICA, AO LONGO DOS ANOS, PARA A ORIGEM CAMPINAS

\begin{tabular}{|c|c|c|c|}
\hline $\begin{array}{l}\text { DIFERENÇA DE } \\
\text { MEDIAS } \\
(\bar{C})\end{array}$ & $\begin{array}{l}\text { DESVIO PADRÃO } \\
\text { DA DIFERENÇA } \\
\text { DE MEEDIAS } \\
\text { (DP }(\bar{C}))\end{array}$ & $\begin{array}{c}\text { INTERVALO DE CON- } \\
\text { FIANÇA DAS } \\
\text { MEDIAS A NIVEL } \\
\text { DE } 99,5 \%\end{array}$ & $\begin{array}{c}\text { RESULTADO DA COMPARA } \\
\text { CCÃO POR BONFERRONI } \\
\text { A NIVEL DE CONFIANÇA } \\
\text { DE } 95 \%\end{array}$ \\
\hline$M_{80}-M_{79}=0,55$ & 0,53 & {$[-1,84 ; 0,74]$} & $M_{80}=M_{79}<M_{77}<$ \\
\hline $\mathrm{M}_{77}-\mathrm{M}_{79}=1,49$ & 0,57 & {$[0,1 ; 2,88]$} & ${ }^{M_{81}}=M_{78}$ \\
\hline$M_{81}-M_{77}=1,25$ & 0,51 & {$[0,01 ; 2,49]$} & \\
\hline $\mathrm{M}_{78}-\mathrm{M}_{81}=0,63$ & 0,43 & {$[-0,41 ; 1,67]$} & \\
\hline
\end{tabular}

NOTAÇÃO: $M_{x y}$ significa nota média do a no $x y$. Por exemplo, $M_{80}$ significa nota média do ano 80 .

A análise do Quadro 11 é análoga. A mesma permite concluir, com um nivel de $95 \%$ de confiança, que a pior performance em Teoria Eco nômica dos candidatos que optaram pela UNICAMP foi em 1980. Os desempenhos foram intermediários nos anos de 1977 e 1979, e melhores nos anos de 1978 e 1981 (ver Gráfico 8). 
Quadro 11

COMPARAÇÕO DAS NOTAS MEDIAS DE TEORIA ECONOMICA;

AO LONGO DOS ANOS, PARA A ONÇ̃̃O LINICAMI!

\begin{tabular}{|c|c|c|c|}
\hline 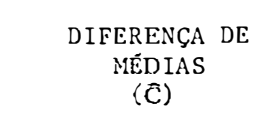 & $\begin{array}{l}\text { DESVIO PADR ÃO } \\
\text { DA DIFERENÇA } \\
\text { DE MEDIAS } \\
\text { (DP (ர)) }\end{array}$ & $\begin{array}{c}\text { INTERVALO DE CON- } \\
\text { FIANÇA DAS } \\
\text { MEDIAS A NIVEL } \\
\text { DE } 99,5 \%\end{array}$ & $\begin{array}{c}\text { RESULTADO DA COSPARA } \\
\text { CSNO P(R BONFERRONII } \\
\text { A NIVEL DE CONFIANCA } \\
\text { DE } 95 \%\end{array}$ \\
\hline $\mathrm{M}_{77}-\mathrm{M}_{80}=1,11$ & 0,36 & {$[0,23 ; 1,99]$} & $M_{80}{ }^{* M_{77}}=M_{79} M_{81}=M_{78}$ \\
\hline$M_{79}-M_{77}=0,54$ & 0,35 & {$[-0,31 ; 1,39]$} & \\
\hline$M_{79}-M_{81}=1,06$ & 0,35 & {$[0,21 ; 1,91]$} & \\
\hline$M_{78}-M_{81}=0,7$ & 0,34 & {$[-0,13 ; 1,53]$} & \\
\hline
\end{tabular}

NOTAÇÃO: My significa nota média do ano $x y$.

nifica nota média do ano 69.

7.2. As notas médias de Métodos Quantitativos,

a o longo dos anos, na ORIGEM Rio

Analisando o Quadro 12 podemos concluir que: os candidatos da ORIGEM Rio tiveram sua pior performance média em Métodos Quantitativos em 1977 e 1981; os resultados foram um pouco melhores em 1978 e 1980 , e a maior nota média ocorreu em 1979.

Quadro 12

COMPARAÇÃO DAS NOTAS MEDIAS DE METODOS.

QUANTITATIVOS, AO LONGO DOS ANOS,

NA ORIGEM RIO

\begin{tabular}{|c|c|c|c|}
\hline $\begin{array}{l}\text { DIFERENÇA DE } \\
\text { MEDIAS } \\
\text { (C) }\end{array}$ & $\begin{array}{l}\text { DESVIO PADRÃO } \\
\text { DA DIFERENÇA } \\
\text { DE MÉDIAS } \\
\text { (DP (C)) }\end{array}$ & $\begin{array}{c}\text { INTERVALO DE CON- } \\
\text { FIANÇA DE } 99,5 \% \\
\text { PARA A DIFERENÇA } \\
\text { DAS MERIAS }\end{array}$ & $\begin{array}{l}\text { RESULTADO DA COMPARA } \\
\text { CẼO POR BONFERR ONI } \\
\text { A NIVEL DE CONFIANÇA } \\
\text { DE } 95 \%\end{array}$ \\
\hline $\begin{array}{l}M_{78}-M_{77}=0,89 \\
M_{81}-M_{77}=0,25 \\
M_{78}-M_{80}=0,26 \\
M_{79}-M_{80}=0,82\end{array}$ & $\begin{array}{l}0,29 \\
0,30 \\
0,28\end{array}$ & 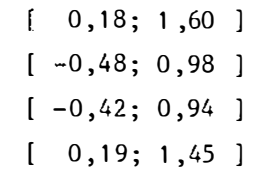 & $M_{77}=M_{81}<M_{78}=M_{80}<M_{79}$ \\
\hline
\end{tabular}

Notaça: My significa nota média do ano xy. Por exemplo, My significa nota média do ano de 1977. 
7.3. Comparaçōes das opcões em Métodos Quantitativos

No caso de Métodos Quantitativos, pelo fato de não existir in terações significativas entre OPÇÃO e os outros dois fatores - con forme mostra o Quadro 9 - podemos fazer comparações globais entre as opções. Assim, os candidatos que optaram pela Universidade de Brasilia e pelo CEDEPLAR/UFMG, tiveram resultados médios piores que aqueles que optaram por Rio, pela UNICAMP ou pela Universidade de são Paulo (ver Quadro 13 ).

Quadro 13

COMPARAÇÃO DAS NOTAS MEDIAS DE METODOS

QUANTITATIVOS, PARA AS

DIVERSAS OPÇOES

\begin{tabular}{|c|c|c|c|}
\hline $\begin{array}{l}\text { DIFERENÇA DAS } \\
\text { NOTAS MEDIAS } \\
\text { ( }(\tilde{C})\end{array}$ & $\begin{array}{l}\text { DESVIO PADRÃO } \\
\text { DA DIFERENÇA } \\
\text { DAS MÉDIAS } \\
(\text { DP }(\bar{C}))\end{array}$ & $\begin{array}{l}\text { INTERVALO DE CON } \\
\text { FIANCA DAS } \\
\text { MEDIAS A NfVEL } \\
\text { DE } 99,5 \%\end{array}$ & $\begin{array}{l}\text { RESULTADO DA COMPARA- } \\
\text { CÁO BONFERRONI A } \\
\text { NFVEL DE } 95 \%\end{array}$ \\
\hline$o_{U n B}-o_{\text {UFMG }}=0,24$ & 0,22 & {$[-0,31 ; 0,75]$} & $\begin{array}{l}O_{\text {UnB }}=0_{\text {UFMG }}<o_{\text {RIO }}= \\
=o_{\text {UNICAMP }}=0_{\text {USP }}\end{array}$ \\
\hline $\mathrm{O}_{\mathrm{RIO}}-\mathrm{O}_{\text {UNICAMP }}=0,27$ & 0,14 & {$[-0,07 ; 0,60]$} & \\
\hline 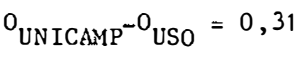 & 0,16 & {$[-0,08 ; 0,70]$} & \\
\hline 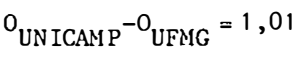 & 0,20 & {$[0,52 ; 1,50]$} & \\
\hline
\end{tabular}

NOTAÇÃO: ${ }_{A B C}$ significa nota média para a opçäo ABC. Por exemplo, OUNICAMP significa nota média dos alunos que fizeram a opsão pela UNICAMP. 


\section{B IBL IOGRAF I A}

1. AguirRe, A. "A Evolução do Nümero de Candidatos e das Notas Mé dias nos Concursos da ANPEC - 1976/1980". Literatura Económica. Rio de Janeiro, 5(1):5-16, jan/fev. 1983.

2. AguirRe, A. "Notas Médias nos Concursos da ANPEC, Classificadas por Local de Prova e por 'Primeira Opcão' -1976/81". Literatura Economica. Rio de Janeiro, 6(4):645-664, out. 1984 .

3. NETER, J. \& WASSERMAN, W. Applied Linear Statisticarl Models. Richard D. Irwin, Inc. Homewood, I11. 1974 . 
